\title{
Class Based Dynamic Priority Scheduling for Uplink to Support M2M Communications in LTE
}

\author{
Mukesh Kumar Giluka ${ }^{\dagger}$, Nitish Rajoria ${ }^{\dagger}$, Ashish C. Kulkarni ${ }^{\dagger \dagger}$, Vanlin Sathya ${ }^{\dagger}$ and Bheemarjuna Reddy Tamma ${ }^{\dagger}$ \\ Indian Institute of Technology Hyderabad, India ${ }^{\dagger}$ \\ PESIT Bangalore, India ${ }^{\dagger \dagger}$ \\ Email: [cs11p1002, cs11m01]@iith.ac.in, ashishkulkarni09@gmail.com, [cs11p1003, tbr]@iith.ac.in
}

\begin{abstract}
Machine-to-Machine (M2M) communication has emerged as a key technology with huge market potential for cellular service providers deploying LTE networks. Addition of enormous number of M2M devices into the cellular networks poses a heavy competition to existing Human-to-Human $(\mathrm{H} 2 \mathrm{H})$ devices for getting radio resources, thereby affecting the performance of the $\mathrm{H} 2 \mathrm{H}$ communications. But, one can not treat all M2M flows as low priority and schedule them after $\mathrm{H} 2 \mathrm{H}$ flows, as there are many M2M applications like healthcare and tracking which are of high importance and delay-intolerant. Hence, there is a need for class based priority scheduling of the traffic of M2M and $\mathrm{H} 2 \mathrm{H}$ sessions in the network. In this paper, we propose a class based dynamic priority scheduling algorithm for uplink transmission of $\mathrm{M} 2 \mathrm{M}$ and $\mathrm{H} 2 \mathrm{H}$ traffic in LTE. The performance of the algorithm is evaluated by various metrics such as $\mathrm{H} 2 \mathrm{H}$ throughput and system throughput and also compared with existing schedulers.
\end{abstract}

\section{INTRODUCTION}

The Internet of things (IoT) [1] can be characterized by interconnecting uniquely identifying objects. Presently, IoT can be realised with the help of an Internet-like-structure. Machine-to-Machine (M2M) communication is an emerging technology which deals with networking part of IoT [1]. Typically, in IoT/M2M applications, M2M devices monitor events (e.g., temperature, inventory level), which is relayed through a wireless communication network infrastructure to an application (software program running on a Server connected to the Internet), that translates the monitored event into meaningful information to be able to take collaborative decisions with limited or no human intervention. Examples include smart transportation, environment monitoring, smart grid, smart healthcare, and fleet management which will entirely transform how humans interact with physical world. According to the predictions [1] by the end of year 2020, approximately 20 billion IoT/M2M devices will be deployed on the planet Earth and offer various noble M2M applications by connecting to the Internet.

Because of ubiquitous coverage and global connectivity, cellular networks can play a major role in the deployment of M2M applications. Cellular networks can be used in two different ways for supporting M2M communications: (a) an $\mathrm{M} 2 \mathrm{M}$ device having embedded cellular radio sends data directly to the M2M server located on Internet through a cellular network. (b) an M2M device having embedded WiFi or ZigBee radio first sends data to an M2M gateway and then gateway forwards data (typically after aggregation) to the
M2M server through a cellular network. Former is called as cellular M2M communication while later is called as capillary M2M communication [1]. In capillary M2M, gateway needs dual radios for supporting connection to cellular and $\mathrm{Wi}$ Fi/ZigBee networks. In this paper, we will deal with only cellular M2M communication scenario.

Presently, cellular networks are optimized for Human-toHuman $(\mathrm{H} 2 \mathrm{H})$ and Human-to-Machine $(\mathrm{H} 2 \mathrm{M})$ communications and in future, these seem to be uninterrupted because of the percentage of revenue contributed by these applications. At the same time, characteristics of $\mathrm{H} 2 \mathrm{H} / \mathrm{H} 2 \mathrm{M}$ communication are different from that of $\mathrm{M} 2 \mathrm{M}$ in terms of traffic volume, uplink to downlink traffic ratio, density of devices in a particular geographical area and mobility. With these differences, supporting M2M communication in cellular networks is a major challenge to the telecom network operators. Among the several challenges to be dealt due to the incorporation of M2M applications in cellular networks, efficient allocation of radio resources between $\mathrm{M} 2 \mathrm{M}$ applications and $\mathrm{H} 2 \mathrm{H} / \mathrm{H} 2 \mathrm{M}$ applications is one of the biggest challenges. Due to the limited available radio spectrum and enormous number of M2M devices (i.e., device running M2M applications), existing resource scheduling algorithms do not scale well. In this work, we propose a Class Based Dynamic Priority (CBDP) scheduling algorithm for uplink (i.e., device $\rightarrow$ base station) communication in LTE systems, in which, based on the priority of the class to which $\mathrm{M} 2 \mathrm{M}$ or $\mathrm{H} 2 \mathrm{H} / \mathrm{H} 2 \mathrm{M}$ application belongs to, the radio resources are allocated.

In LTE (Long Term Evolution) networks [2], bandwidth resources are allocated in terms of Resource Blocks (RBs). $\mathrm{A} \mathrm{RB}$ is a smallest unit of bandwidth resource to be allocated to users. In LTE, time is measured in terms of frames. A frame consists of 10 subframes. Duration of a subframe is $1 \mathrm{~ms}$. RBs are allocated by the scheduler for a TTI (Transmission time interval) where TTI is the duration of a subframe.

Rest of the paper is organized as follows: In Sections II, related work is discussed. In Section III, proposed work has been explained. Simulation setup and performance evaluation of the algorithm are given in Section IV and finally, Section V concludes the paper.

\section{RELATED WORK}

In this section, we review existing works addressing scheduling issues due to incorporation of M2M in LTE sys- 
tems. In [3], all the applications are divided into four classes. For each class, an utility function is defined where user utility is a function of achievable data rate. The main objective of their scheduling algorithm is to maximize the aggregate utility of the function so that aggregate throughput can also be maximized. But, fairness and priority of a device are not taken into account during scheduling process. If a device has very less achievable data rate with respect to some RB then this RB will not be allocated to that device because it will not lead to increase in the aggregate utility. At the same time, if a device is running a delay-intolerant application but assigning a $\mathrm{RB}$ is not maximizing the utility then the device may remain unallocated. In [4], in order to distribute radio resources between $\mathrm{H} 2 \mathrm{H}$ and $\mathrm{M} 2 \mathrm{M}$ flows two algorithms have been proposed. $\mathrm{H} 2 \mathrm{H}$ flows are given high priority over M2M flows in both algorithms. So, resources are allocated first to all $\mathrm{H} 2 \mathrm{H}$ flows and if remaining, resources are allocated to M2M flows. The first algorithm gives higher priority to the signal to interference and noise ratio (SINR) value at a RB with respect to $\mathrm{M} 2 \mathrm{M}$ device, in comparison to delay tolerance level during the allocation of RBs to M2M devices. The second algorithm gives higher priority to delay tolerance level than the SINR value. The main drawback of these algorithms is that they do not allocate RBs to M2M devices based on the applications they are running. It does not differentiate the delay tolerant and delay intolerant M2M devices and therefore efficient allocation of RBs in not done.

In [5], authors define a cluster of M2M devices based on the parameters: packet arrival rate and maximum tolerable jitter. An M2M device belongs to a cluster if both the device and cluster have identical values of these parameters. A cluster with larger packet arrival rate has high priority. Depending on the traffic rate and priority of cluster, a fixed access grant time interval will be allocated to clusters. The main drawback of the paper is that it consider traffic arrival rate as constant while in reality, M2M traffic can be random in nature. In [6], authors have attempted to remove the drawbacks of [5] but they did not consider the cases when M2M applications should be given more preferences over $\mathrm{H} 2 \mathrm{H}$ applications.

In this paper, we have proposed a scheduling algorithm which considers delay tolerance and minimum guaranteed bit rate of applications and preference of $\mathrm{H} 2 \mathrm{H}$ flows over $\mathrm{M} 2 \mathrm{M}$ flows to schedule RBs. Apart from this, we propose variable chunk size based method for contiguous allocation of RBs to an user for uplink transmission.

\section{PROPOSED WORK}

Different applications have different QoS requirements in nature. In [7], authors have classified all applications (both $\mathrm{H} 2 \mathrm{H}$ and $\mathrm{M} 2 \mathrm{M}$ ) based on the parameters delay tolerance/delay sensitivity, accuracy and priority. Except the parameter delay tolerance/delay sensitivity, other parameters can be taken care by higher layers and can be ensured by mutual communication of end devices. But as far as scheduling of radio resources in wireless medium is concerned, delay sensitivity of the application is the most important parameter to deal with. With respect to a radio resource scheduler, types of applications can be described as following: (1) Type 1: Applications that send very small amount of data but delay intolerant in nature. For example, emergency alerting, signalling messages. (2) Type 2: Applications that need a minimum guaranteed bit rate (MGBR). These application have a delay tolerance time (DTT) within which a certain amount of data must be scheduled by the scheduler to meet the MGBR. For example, streaming video, VOIP, and IP enabled surveillance cameras. (3) Type 3: Applications that have bulk data to send but are delay tolerant in nature. For example, file downloads. (4) Type 4: Applications that send very small amount of data and delay tolerant in nature. For example, environment monitoring M2M applications.

In this paper, we propose delay aware radio resource scheduler to support M2M traffic without affecting or least affecting $\mathrm{H} 2 \mathrm{H}$ traffic. The goal of designing such a scheduler is to address following issues: (1) to support delay intolerant $\mathrm{H} 2 \mathrm{H}$ or M2M flows. (2) to ensure MGBR of type 2 applications discussed in last paragraph. (3) to give preference to $\mathrm{H} 2 \mathrm{H}$ flows over M2M flows in case of similar QoS requirements. (4) to postpone the scheduling of delay tolerant flows if enough radio resources are not available.

In order to achieve above goals, we first classify all flows based on their remaining time to serve (RTTS) within which the flow must be served by the scheduler to meet the DTT of the flow. If DTT of a flow is $30 \mathrm{~ms}$ then initially its RTTS will also be $30 \mathrm{~ms}$ but if the flow does not get served till $5 \mathrm{~ms}$ then its RTTS will become $25 \mathrm{~ms}$. All delay intolerant applications (type 1) are kept in class 1 while all delay tolerant applications (type 3 and type 4) are kept in class $n$ (last class). Other applications or flows are kept between class 2 to class $n$ 1. Except class $n$, a class is defined by a range of RTTSs (say [20-30] ms). If RTTS of a flow lies within this range, then it means that the flow belong to that class. So, if RTTS of a flow is $25 \mathrm{~ms}$ then it lies in this class. A class is given higher priority than other class if higher value of its RTTS range is lesser than the lower value of RTTS range of other class. So, if class 1 has RTTS range [10-20] ms and class 2 has RTTS range [20-30] ms then priority of class 1 is higher than class 2 . A class with high priority will be served first by the scheduler than a low priority class. If a flow belonging to low priority class is not served within the RTTS range of the class then the flow will automatically get shifted to next high priority class. In a TTI, if a flow is not being served then its RTTS value decreases by $1 \mathrm{~ms}$. Since type 3 and type 4 applications are delay tolerant in nature, in class $n$, all delay tolerant applications are kept. These applications or flows will not have any RTTS value. So, a flow belonging to class $n$ will remain in the same class.

Algorithm 1 lists out the proposed Class Based Dynamic Priority (CBDP) scheduling algorithm. The algorithm running at the base station (BS) allocates RBs to flows on per TTI basis. The algorithm takes following parameters as input: (i) A set of all flows which are waiting for uplink RB scheduling by the BS. All flows will have RTTS except flows generated 


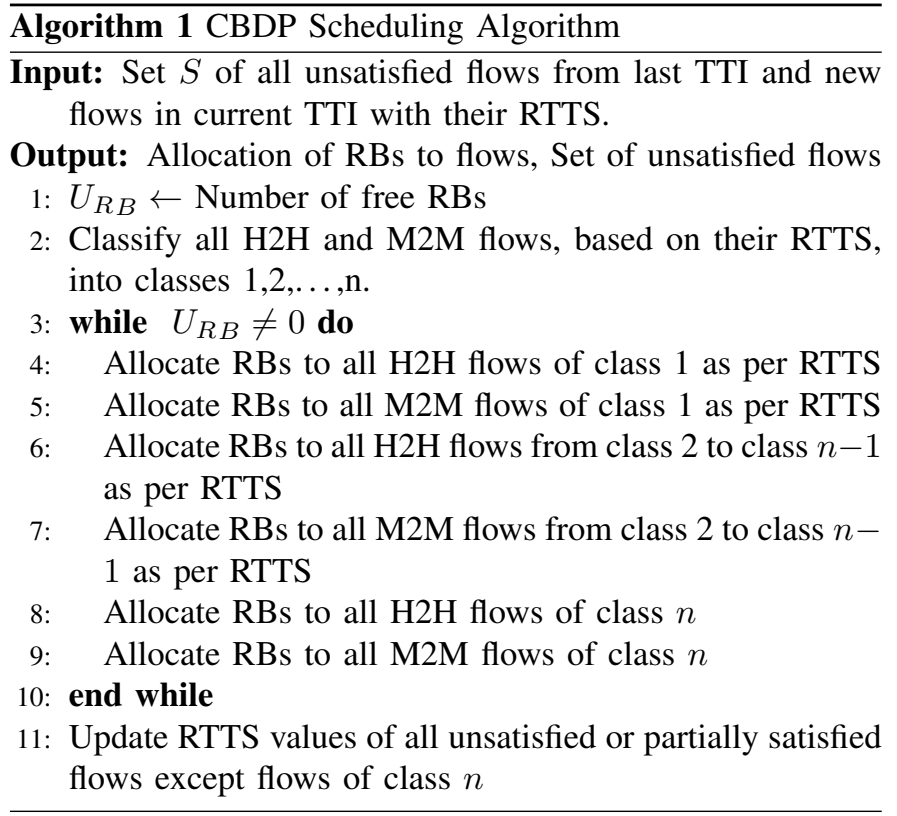

by type 3 and type 4 applications. (ii) Range of RTTS of each class (except class $n$ ). With the help of these parameters, the scheduler assigns classes to all flows in each TTI (before scheduling the RBs). A class not only contains those flows which originally belong to that class but also contains those flows who originally belong to some low priority classes but because of dynamic priority concept, after sufficient decrement in their RTTS values (because of not being served for some TTIs), they moved to this class. In a class, a flow having minimum RTTS will be served first.

The algorithm first serves all the flows of class 1 so that delay intolerant applications can meet their delay constraints. In class 1 , all $\mathrm{H} 2 \mathrm{H}$ flows are served before $\mathrm{M} 2 \mathrm{M}$ flows in the ascending order of RTTS values. The motivation behind giving preference to $\mathrm{H} 2 \mathrm{H}$ flows is not to affect them because of M2M flows of same QoS requirements. Now, from class 2 to class $n-1$, all $\mathrm{H} 2 \mathrm{H}$ flows will be served first. After serving $\mathrm{H} 2 \mathrm{H}$ flows, again from class 2 to class $n-1$ all M2M flows will be served. Since, a flow (M2M or $\mathrm{H} 2 \mathrm{H})$ belonging to any class in between class 2 to class $n-1$ can tolerate some amount of delay in allocation of resources, in order to give preference over M2M flows, all $\mathrm{H} 2 \mathrm{H}$ flows belonging to class 2 to $n-1$ are allocated RBs first and then allocation for M2M flows takesplace.

The scheduler will schedule at least $M G B R * D T T$ amount of data in DTT for all flows belonging to class 2 to class $n-1$. If $\mathrm{RBs}$ are available after serving all classes, more can be allocated to flows of these classes. For the flows of class $n$, neither they have any delay constraint like class 1 flows nor they have to meet any MGBR. So, the scheduler allocates RBs to these flows in best-effort manner.

Figure 1 shows an example of CBDP scheduling order for four classes of $\mathrm{H} 2 \mathrm{H}$ and $\mathrm{M} 2 \mathrm{M}$ flows in the network. Here, class 1 contains flows of type 1 applications. Class 2 and class 3 contain flows of type 2 applications. Class 4 contains flows of type 3 and type 4 applications. The arrow shows the order in which scheduler allocates RBs to flows belonging to various classes of $\mathrm{M} 2 \mathrm{M}$ and $\mathrm{H} 2 \mathrm{H}$ flows.

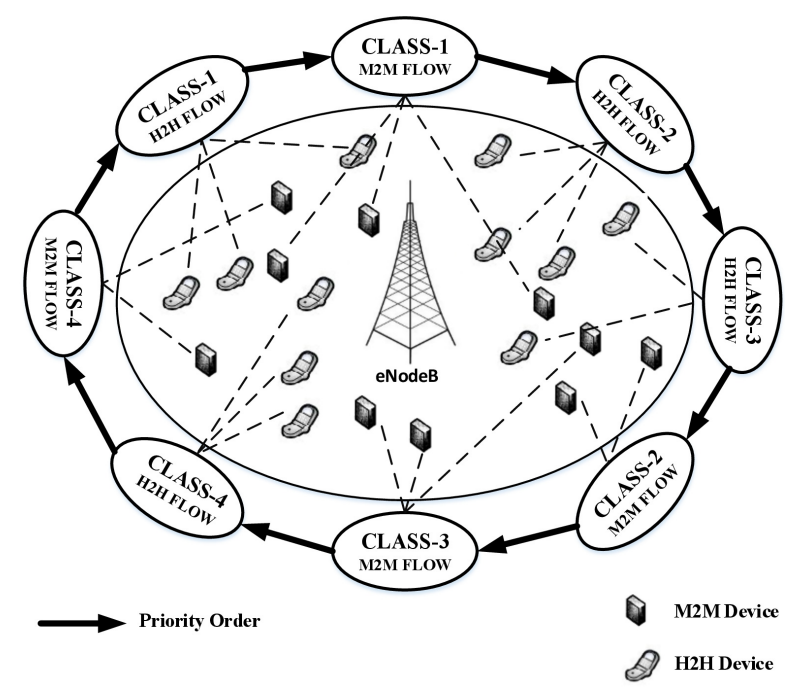

Fig. 1. An example of CBDP scheduling order for four classes of $\mathrm{H} 2 \mathrm{H}$ and M2M flows

\section{A. Contiguity Constraint for Uplink}

The major constraint with LTE uplink resource scheduling is that the number of RBs assigned to a flow must be contiguous because of Single-carrier Frequency Division Multiple Access (SC-FDMA)[8] scheme for uplink channel access. Maximization of throughput with the constraint of contiguous $\mathrm{RB}$ allocation is a NP-Hard problem. Hence, in this paper, we propose variable chunk size based uplink resource allocation heuristic scheme where a flow is assigned best available chunk of contiguous RBs to upload its data. Size of a chunk is the number of contiguous RBs required to send the desired amount of data. Size of a chunk, to meet demand of a flow, will be different for different sets of RBs because of change in CQI (channel quality information) with respect to RBs and user location. The Algorithm 2, the Contiguity Constraint Algorithm (CCA), is used to ensure the contiguous allocation of RBs to requests generated by flows in Algorithm 1. Algorithm 2 is called by Algorithm 1 in steps $4-9$. In CCA, $R$ denotes a set of unallocated, contiguous RBs and $N E E D$ denotes a two dimensional matrix where $(N E E D)_{i j}$ is the chunk size needed to meet the demand of flow $i$, if first RB of the chunk is $j^{t h} \mathrm{RB}$ of the set $R$. So, row $i$ contains the value of required chunk sizes to send data of flow $i$ with respect to each RB (i.e., as a first $\mathrm{RB}$ of the chunk) of set $R$. Now the algorithm will choose a chunk of minimum size with which the flow $i$ can send its data. If the chosen chunk is already assigned to some request then the algorithm will choose next minimum 
size chunk and will check its availability. If the chosen chunk is free then it will be assigned to flow $i$ and RBs of the chunk will be marked as allocated in the set $R$. After allocating RBs to flow $i$, the algorithm will move to schedule $(i+1)^{t h}$ flow.

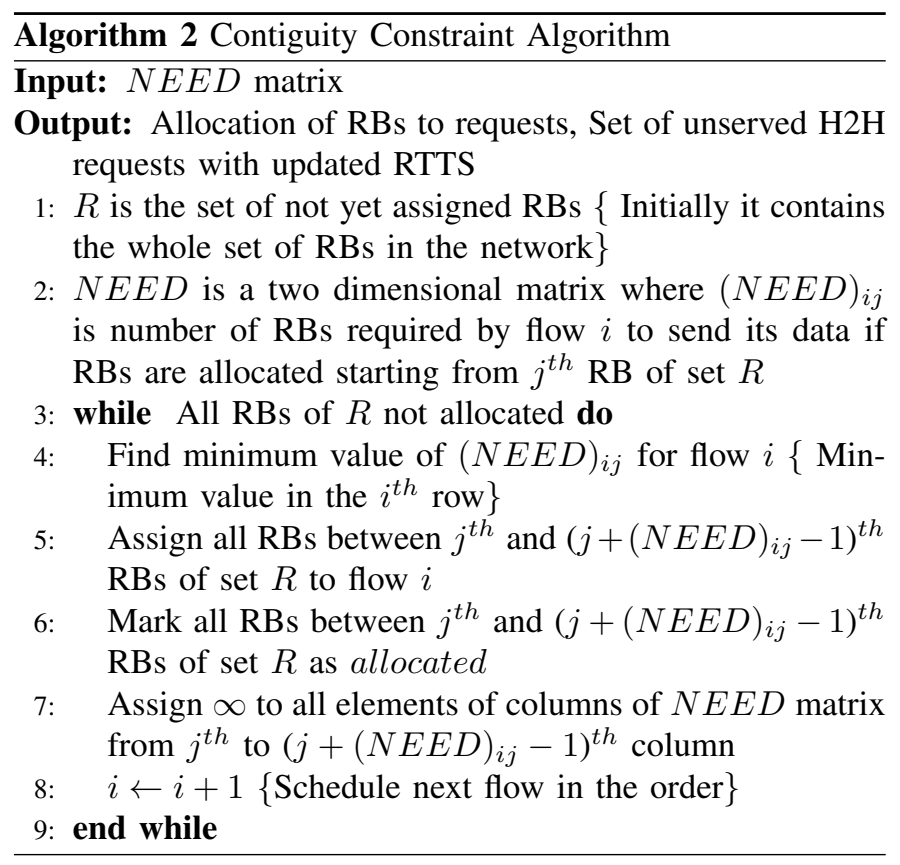

\section{Simulation Results AND ANALYSis}

In this section, the performance of proposed CBDP algorithm is evaluated using system level simulations in NS-3.16 network simulator [9]. The simulation parameters are specified in Table I and parameters not specified here are assumed to be the default ones mentioned in 3GPP specifications [2]. The performance evaluation is done with a single-cell environment with an omni-directional antenna. The bandwidth on the uplink is $10 \mathrm{MHz}$, sub-divided into $50 \mathrm{RBs}$. All the devices are randomly located in a radius of $1 \mathrm{~km}$ using Random Disc Position Allocator Model. Table II and Table III show the simulation parameters of $\mathrm{H} 2 \mathrm{H}$ and $\mathrm{M} 2 \mathrm{M}$ flows, respectively.

TABLE I

SiMULATION SETUP

\begin{tabular}{|c|c|}
\hline Simulator & NS-3.16 \\
\hline System Bandwidth & $10 \mathrm{MHz}$ \\
\hline Cellular Layout & Single-Cell with Omni-directional Antenna \\
\hline No. of RBs in a TTI & 50 \\
\hline TTI Duration & $1 \mathrm{~ms}$ \\
\hline Total No. of Devices & 80 \\
\hline UE-eNodeB Min. Distance & $10 \mathrm{~m}$ \\
\hline Simulation Time & $1 \mathrm{sec}$ \\
\hline
\end{tabular}

We have compared the performance of proposed CBDP algorithm with proportional fair (PF) and round-robin (RR) scheduling algorithms. Initially, we have analyzed how throughput of $\mathrm{H} 2 \mathrm{H}$ flows get affected with the incorporation of M2M flows in the network. For that we have compared
TABLE II

Simulation PARAMETERS FOR H2H Flows

\begin{tabular}{|c|c|c|c|c|c|}
\hline \hline \multicolumn{2}{|c|}{ Class } & \multicolumn{4}{c|}{ H2H } \\
\hline $\begin{array}{l}\text { Class } \\
\text { Number }\end{array}$ & $\begin{array}{l}\text { Range } \\
\text { of RTTS }\end{array}$ & Application & MGBR & DTT & $\begin{array}{l}\% \text { of } \\
\text { H2H } \\
\text { Flows }\end{array}$ \\
\hline 1 & $0-20 \mathrm{~ms}$ & IMS Signaling & - & $10 \mathrm{~ms}$ & $10 \%$ \\
\hline 2 & $20-100 \mathrm{~ms}$ & Voice, Gaming & $10 \mathrm{kbps}, 10 \mathrm{kbps}$ & $25 \mathrm{~ms}, 50 \mathrm{~ms}$ & $30 \%, 20 \%$ \\
\hline 3 & $100-500 \mathrm{~ms}$ & Video & $100 \mathrm{kbps}$ & $110 \mathrm{~ms}$ & $20 \%$ \\
\hline 4 & Rest & Web Browsing & - & - & $20 \%$ \\
\hline \hline
\end{tabular}

TABLE III

Simulation PARAMETERS FOR M2M FLOWS

\begin{tabular}{|c|c|c|c|c|c|}
\hline \hline \multicolumn{2}{|c|}{ Class } & \multicolumn{4}{|c|}{ M2M } \\
\hline $\begin{array}{l}\text { Class } \\
\text { Number }\end{array}$ & $\begin{array}{l}\text { Range } \\
\text { of RTTS }\end{array}$ & Application & MGBR & DTT & $\begin{array}{l}\text { M2M } \\
\text { Flows }\end{array}$ \\
\hline 1 & $0-20 \mathrm{~ms}$ & Emergency & - & $10 \mathrm{~ms}$ & $10 \%$ \\
\hline 2 & $20-100 \mathrm{~ms}$ & - & - & - & - \\
\hline 3 & $100-500 \mathrm{~ms}$ & Surveilance Camera & $100 \mathrm{kbps}$ & $110 \mathrm{~ms}$ & $15 \%$ \\
\hline 4 & Rest & Regular Monitoring & - & - & $75 \%$ \\
\hline \hline
\end{tabular}

the throughput of $\mathrm{H} 2 \mathrm{H}$ flows in CBDP and PF schedulers. After that we evaluated class wise throughput of all $\mathrm{H} 2 \mathrm{H}$ and M2M flows for the proposed CBDP scheduler. At the end, we compared the system throughput or aggregate throughput with respect to all three schedulers.

In Figure 2, we have analyzed how $\mathrm{H} 2 \mathrm{H}$ flows are getting affected in terms of aggregate throughputs when number of $\mathrm{M} 2 \mathrm{M}$ flows increase. Here, we have taken $20 \mathrm{H} 2 \mathrm{H}$ flows for $\mathrm{CBDP}$ and PF schedulers. Number of M2M flows is increased from 0 to 60 . We can observe that CBDP algorithm performs better in comparison to PF algorithm. This is because of the fact that in case of CBDP, H2H flows are served first and then M2M flows are served while in case of PF scheduler, resources are shared between $\mathrm{H} 2 \mathrm{H}$ and $\mathrm{M} 2 \mathrm{M}$ flows equally which results into the degradation of throughput of $\mathrm{H} 2 \mathrm{H}$ flows.

In Figure 3, classwise throughputs for different number of

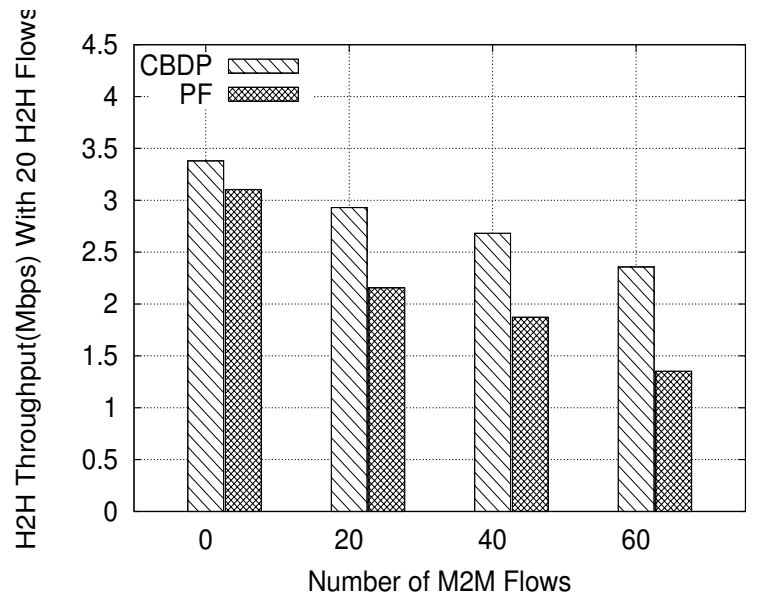

Fig. 2. Comparison of aggregate throughputs for $\mathrm{H} 2 \mathrm{H}$ flows 
flows have been shown for proposed CBDP scheduler. A class may contain both $\mathrm{H} 2 \mathrm{H}$ and M2M flows. Since, in class 1 both $\mathrm{H} 2 \mathrm{H}$ and M2M flows have very small amount of data to send, throughput of class 1 is not visible in the graph. But practically, all flows of class 1 are satisfied. We can observe from the graph that throughputs of class 2 and class 3 increase with the increasing of total number of flows. But throughput of class 4 increases up to 40 flows but after that it decreases. Since, in class 2 and class 3 we are ensuring a minimum guaranteed bit rate, so throughput increases while in class 4 , flows are served in best effort manner. Since, class 4 contains all delay tolerant flows, so they are scheduled later when RBs are free due to lack of higher class traffic.

In Figure 4, aggregate throughputs of all flows $(\mathrm{H} 2 \mathrm{H}$ and

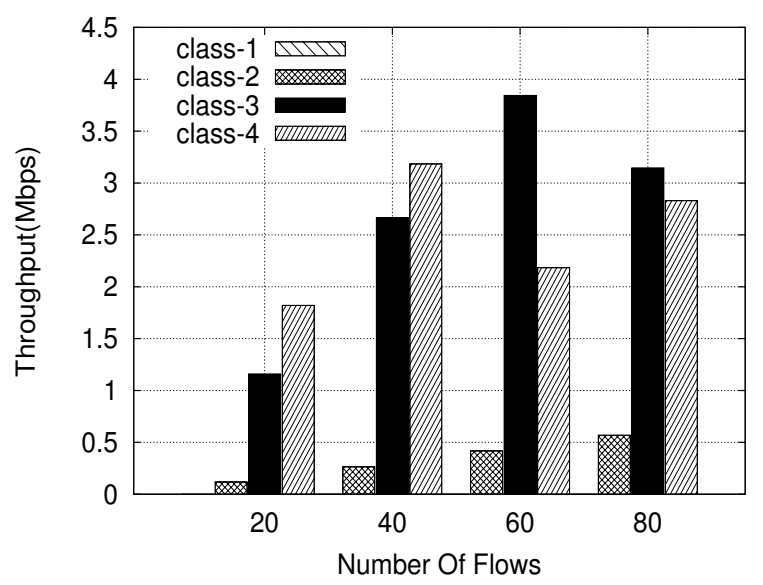

Fig. 3. Comparison of classwise aggregate throughputs in CBDP scheduler

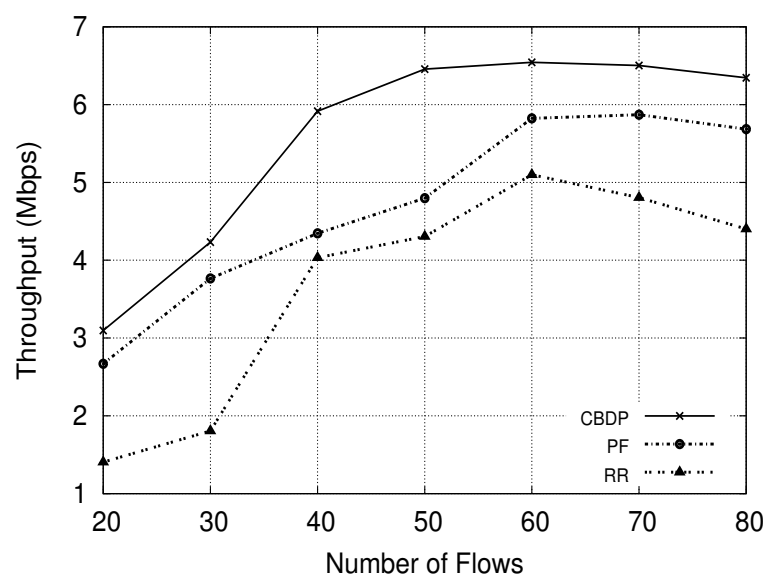

Fig. 4. Comparison of Sytem throughput of CBDP, PF and RR schedulers

M2M both) with respect to all schedulers considered have been shown. Here, the CBDP algorithm performs better than $\mathrm{PF}$. The main reason for this is that in the case of PF, to ensure the contiguous allocation of RBs, a flow is assigned a chunk of constant size. So, it is possible that some of RBs in a chunk will remain unallocated if data to be sent by the flow is finished after utilizing only a some of RBs of the chunk. But in the case of CBDP algorithm, chunk with variable size is allocated to ensure the contiguity of RBs. Because of that same amount of data can be sent with less number of RBs and therefore efficient RB utilization is achieved. Quantatively we can say that, depending on total number of flows, throughput shown by CBDP scheduler is $27-100 \%$ more than RR scheduler while in case of PF scheduler, it is $7-40 \%$. From above results, we can conclude that CBDP algorithm performs best in terms of supporting M2M devices with least affecting $\mathrm{H} 2 \mathrm{H}$ devices.

\section{CONCLUSION}

Support of IoT/M2M applications in existing cellular networking technologies is an interesting and challenging problem. If proper radio resource allocation schemes are not employed, these M2M applications could drastically impact the performance of $\mathrm{H} 2 \mathrm{H} / \mathrm{H} 2 \mathrm{M}$ applications and the telecom operators may end up facing customer churn. The main goal of the proposed class based dynamic priority (CBDP) scheduling for uplink, was to support M2M communications while least affecting $\mathrm{H} 2 \mathrm{H}$ communications. We compared the proposed CBDP algorithm with PF and RR scheduling algorithms and found that even in presence of M2M flows, CBDP algorithm not only satisfied the QoS requirements of $\mathrm{H} 2 \mathrm{H}$ flows but also satisfied QoS requirements of M2M flows.

As a future work, we are planning to simulate the algorithm for thousand M2M flows with more specific M2M applications. Apart from this, we will calculate the extra signalling overhead incurred by the algorithms.

\section{ACKNOWLEDGMENT}

This work was supported by the Deity, Govt of India (Grant No. 13(6)/2010CC\&BT).

\section{REFERENCES}

[1] Min Chen, Jiafu Wan and Fang Li, "Machine-to-Machine Communications: Architectures, Standards and Applications", KSII Transactions on Internet and Information Systems, vol. 6, no. 2, February 2012.

[2] 3GPP TS 36.211 V8.7.0, "Evolved Universal Terrestrial Radio Access (E-UTRA); Physical Channels and Modulation (Release 8)", June 2009.

[3] Kan Zheng, Fanglong Hu, Wenbo Wang, Wei Xiang and Mischa Dohler, "Radio Resource Allocation in LTE-Advanced Cellular Networks with M2M Communications", IEEE Communications Magazine, vol. 50, no. 7, pp. 184-192, July 2012.

[4] Athanasios S. Lioumpas and Angeliki Alexiou, "Uplink Scheduling for Machine-to-Machine Communications in LTE-Based Cellular Systems", in Proc. of IEEE GLOBECOM Workshop on Machine-to-Machine Communication, December 2011.

[5] Shao-Yu Lien, Kwang-Cheng Chen and Yonghua Lin, "Toward Ubiquitous Massive Accesses in 3GPP Machine-to-Machine Communications", IEEE Communications Magazine, vol. 49, no. 4, pp. 66-74, April 2011.

[6] Antonis G. Gotsis, Athanasios S. Lioumpas and Angeliki Alexiou, "Analytical Modeling and Performance Evaluation of Realistic TimeControlled M2M Scheduling over LTE Cellular Networks", European Transactions on Telecommunications, vol. 24 , no. 4, pp. 378-388, June 2013.

[7] Rongduo Liu, Wei Wu, Hao Zhu, Dacheng Yang, "M2M-Oriented QoS Categorization in Cellular Network", in Proc. of WiCOM 2011, September 2011.

[8] Jeongchan Kim, Donggeun Kim, Youngnam Han, "Proportional Fair Scheduling Algorithm for SC-FDMA in LTE Uplink", in Proc. of IEEE Global Communications Conference (GLOBECOM), December 2012.

[9] Network Simulator. Available online at: "http://www.nsnam.org". 